\title{
Compliance and emission trading rules for asymmetric emission uncertainty estimates
}

\author{
Zbigniew Nahorski · Joanna Horabik
}

Received: 5 January 2009 / Accepted: 15 June 2010 / Published online: 14 July 2010

(C) The Author(s) 2010. This article is published with open access at Springerlink.com

\begin{abstract}
Greenhouse gases emission inventories are computed with rather low precision. Moreover, their uncertainty distributions may be asymmetric. This should be accounted for in the compliance and trading rules. In this paper we model the uncertainty of inventories as intervals or using fuzzy numbers. The latter allows us to better shape the uncertainty distributions. The compliance and emission trading rules obtained generalize the results for the symmetric uncertainty distributions that were considered in the earlier papers by the present authors (Nahorski et al., Water Air \& Soil Pollution. Focus 7(4-5):539-558, 2007; Nahorski and Horabik, 2007, J Energy Eng 134(2):47-52, 2008). However, unlike in the symmetric distribution, in the asymmetric fuzzy case it is necessary to apply approximations because of nonlinearities in the formulas. The final conclusion is that the interval uncertainty rules can be applied, but with a much higher substitutional noncompliance risk, which is a parameter of the rules.
\end{abstract}

\section{Introduction}

Emission of greenhouse gases is a basic element of the climate change models. See, for example, Stern (2007) where results are presented in probabilistic terms. However, greenhouse gas inventories estimates are not calculated exactly. Possible error magnitudes depend on the types of gas considered, activities involved, and countries, ranging from a few to over 100 percent. Moreover, distributions of errors for different gases as well as for national inventories may be asymmetric (Ramirez

Electronic supplementary material The online version of this article (doi:10.1007/s10584-010-9916-4) contains supplementary material, which is available to authorized users.

Z. Nahorski $(\varangle) \cdot$ J. Horabik

Systems Research Institute, Polish Academy of Sciences,

Newelska 6, 01-447 Warsaw, Poland

e-mail: Zbigniew.Nahorski@ibspan.waw.pl 
et al. 2006; Winiwarter and Muik 2007). The methods of checking compliance and, particularly, establishing rules for emission trading proposed to date for the uncertain inventories (Jonas et al. 2010; Jonas and Nilsson 2007; Nahorski et al. 2007; Nahorski and Horabik 2008) concern only the symmetric distributions and mainly the interval uncertainty models.

In Nahorski et al. (2007) the compliance and trading rules were considered for the interval uncertainties of emissions. In order to have a high enough likelihood of fulfilling the compliance, lower limit of reductions were required (undershooting), and an appropriate recalculation of the traded emissions needed to be performed. However, the interval uncertainty approach provides too conservative a reduction of limits and a recalculation of traded emissions. Although the stochastic case may be useful for the determination of new compliance rule, see also Gillenwater et al. (2007), only a complicated formula for recalculation of the traded emissions has been provided (Nahorski et al. 2007), which is practically useless because it is valid only for uncorrelated inventories. In this paper a fuzzy uncertainty is considered. The fuzzy set calculus basically inherits the rules from the interval calculus and thereby provides simpler calculations than that for the stochastic variables. At the same time the fuzzy variables may be shaped to have distributions that are more concentrated around observed values than in the interval case, where the information on distribution is lost. Thus, it can better approximate the real distributions. This paper also deals with the asymmetric cases, aiming to improve the precision of assessments as to whether the given emission limits or reductions are satisfied, and being able to guarantee (with a small prescribed risk) that this limit or reduction has been fulfilled in emission trading among parties and in other possible flexible mechanisms under the Kyoto Protocol. Improved precision, as compared with the interval case, means lower compliance costs and more reliable estimates of inventories for the climate change models.

We derive in this paper a new formula for recalculation of the trading quantities for the fuzzy and symmetric distributions, which is a generalization of that used for the interval approach. To obtain an analogous formula for the asymmetric fuzzy case, an approximation is required. The one proposed in this paper is a generalization of those for both the symmetric fuzzy case and the asymmetric interval approach.

Summing up, we derive here new rules for checking compliance and for emission trading, for asymmetric fuzzy distributions. They are generalizations of the rules presented in Nahorski et al. (2007) and Nahorski and Horabik (2008) for symmetric distributions and interval uncertainty and they reduce to them as special instances provided that appropriate parameters are taken. Comparison of the rules obtained for the fuzzy approach with those for the interval approach shows that the latter can be used equivalently, but with a much bigger substitutional parameter than originally designed for the noncompliance risk.

In Section 2 we formulate the problem and introduce some basic notation. Then, in Section 3, we deal with the asymmetric interval uncertainty and we derive conditions for checking compliance and formulas for so-called effective emissions, which can be directly traded, without taking into account the emission uncertainty. In Section 4 a family of fuzzy numbers is introduced. These are used to model the full inventory uncertainty and form the basis for derivations of generalized compliance and emission trading rules. These rules are compared with the interval approach rules, and their equivalence in applications considered in the paper, for appropriately chosen parameters, is shown. Section 6 concludes. 


\section{Problem formulation}

Two systems for reducing greenhouse gases emissions have been applied. One, called cap and trade, as, for example, in the European Trade System, where the limits on emissions from chosen activities are distributed among member countries in the first stage and then finally between companies within the European Union. The problem here is to check, if $L$, the given emission limit for the company, expressed as emission permit, has not been exceeded, that is, if

$$
x \leq L
$$

where $x$ is the real, unknown emission of a party in a given year. Unfortunately, $x$ is not known exactly, as only its available estimate of the emission $\hat{x}$ can be calculated. The estimate of the total emissions by a party is calculated from an inventory of emissions from every contributing activity, including absorption by sinks. These are, however, highly uncertain, see (Winiwarter 2004; Monni et al. 2007). Moreover, uncertainties of inventories $\hat{x}$ differ among different activities both in terms of the range and distributions. Another system used under the Kyoto Protocol requires each participating country to reduce a pre-specified percentage of its base year emissions within the given period (from 1990 to 2008-2012 for most countries), although some countries are granted an opportunity to stabilize emissions at the base year level or even to increase its emissions in a limited way. Three so-called flexible mechanisms are connected with the Kyoto Protocol. These are: Joint Implementation, Clean Development Mechanism, and Permit Trading. All are related to buying the emissions saved by other parties. In all these cases, the problem is to check to see if the declared reduction has actually been achieved.

With emission reductions, the compliance checking is slightly more complicated than in the cap-and-trade system because the limit referred to is also uncertain. This leads to the problem of comparing two uncertain values. Here, however, this problem will be transformed to the form similar to (1), that is to the comparison of uncertain value with the exactly known limit. Let us denote by $\delta$ the fraction of the party's emissions to be reduced. The value of $\delta$ may be negative, for parties required to limit their emission increase. Denoting by $x_{b}$ the basic emission and by $x_{c}$ the emission to be checked, the following inequality should be satisfied

$$
x_{c}-(1-\delta) x_{b} \leq 0
$$

This inequality has the same form as $(1)$, with the inspected variable $x_{c}-(1-\delta) x_{b}$ and the limit $L=0$. Similarly as earlier, neither $x_{c}$ nor $x_{b}$ are known precisely enough. Thus, only the difference in estimates can be calculated

$$
\hat{x}_{c}-(1-\delta) \hat{x}_{b}
$$

where both $\hat{x}_{c}$ and $\hat{x}_{b}$ are known inaccurately. In the Kyoto Protocol context, $x_{b}$ is the emission in the basic year and $x_{c}$ the emission in the compliance period. We are not, however, interested here in reference and compliance times, but only in the values to be compared.

Moreover, the emission estimate of a party may be modified by selling or buying uncertain emissions, which adds to the final uncertainty on the left hand side. These problems are discussed in the sequel using two models of uncertainty: interval and fuzzy. 


\section{Interval uncertainty}

Material in this section is a generalization of the results for the symmetric intervals given in Nahorski et al. (2007). The methodological concept is the same, but the results differ because of changed assumptions, although they do reduce to the previous results, when the symmetric intervals are considered in the equations. The derivations in this section are fundamental for the rest of the material and are therefore presented in a fairly complete way, even if they are more or less straight generalizations of the formulas for the symmetric intervals.

\subsection{Compliance}

Let us denote the lower spread of the uncertainty interval by $d^{l}$ and the upper spread by $d^{u}$. Then, the real basic emission $x_{b}$ and the real checked emission $x_{c}$ are situated in the intervals

$$
x_{b} \in\left[\hat{x}_{b}-d_{b}^{l}, \hat{x}_{b}+d_{b}^{u}\right], \quad x_{c} \in\left[\hat{x}_{c}-d_{c}^{l}, \hat{x}_{c}+d_{c}^{u}\right]
$$

Known limit We start with the simpler case of the limit $L$ which is known exactly. To be completely sure that a party (typically a company) fulfills the limit, its emission inventory should satisfy the following condition, see Fig. 1a.

$$
\hat{x}_{c}+d_{c}^{u} \leq L
$$

As the bounds can be quite large, a weaker condition will be used, see Nahorski et al. (2007). A party is compliant with the risk $\alpha$ if its emission inventory satisfies the condition

$$
\hat{x}_{c}+d_{c}^{u} \leq L+\alpha\left(d_{c}^{l}+d_{c}^{u}\right)
$$

The risk is here understood as a likelihood that the party may not fulfill the agreed obligation regarding the emission limit or reduction because of the uncertainty of the emission inventory.

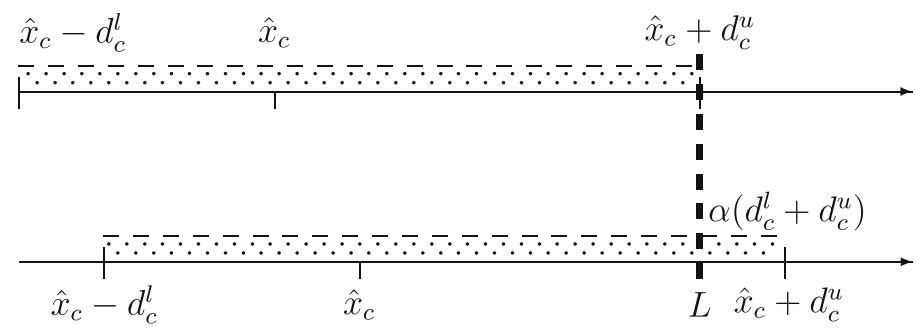

Fig. 1 Full compliance (a) and compliance with risk $\alpha(\mathbf{b})$ in the interval uncertainty approach for the known limit case 
Condition (5) means that the $\alpha$ th part of the party's emission estimate (inventory) uncertainty interval is allowed to lie above the limit $L$, see Fig. 1b. After some algebraic manipulations the condition (5) can be also written in the following form

$$
\hat{x}_{c}+\left[1-\left(1+\frac{d_{c}^{l}}{d_{c}^{u}}\right) \alpha\right] d_{c}^{u} \leq L
$$

The above condition shows that a part of the upper spread of the uncertainty interval is added to the emission estimate before compliance is checked. This can be also interpreted to mean that an unreported emission, due to uncertainty, is included in the condition to reduce the risk of non-compliance.

For the symmetric interval $d_{c}^{l}=d_{c}^{u}=d_{c}$ the condition (6) takes the form

$$
\hat{x}_{c}+(1-2 \alpha) d_{c} \leq L
$$

which has been derived in Nahorski and Horabik (2008).

Emission reduction A more difficult case of checking an emission reduction, when both the checked and the basic emission are uncertain, will be transformed to the problem of a known limit by considering the difference of the checked and reduced emissions, as mentioned earlier. Using the interval calculus rules, we get

$$
x_{c}-(1-\delta) x_{b} \in\left[D \hat{x}-d_{b c}^{l}, D \hat{x}+d_{b c}^{u}\right]
$$

where

$$
D \hat{x}=\hat{x}_{c}-(1-\delta) \hat{x}_{b}
$$

and the lower and upper spreads are

$$
d_{b c}^{l}=d_{c}^{l}+(1-\delta) d_{b}^{u}, \quad d_{b c}^{u}=d_{c}^{u}+(1-\delta) d_{b}^{l}
$$

However, the inventories $\hat{x}_{b}$ and $\hat{x}_{c}$ are dependent and the values of $d_{b c}^{l}$ and $d_{b c}^{u}$ are usually much smaller than those resulting from the above expression. Nahorski et al. (2007) proposed to take this into account by modification of the formulas (8) to

$$
\begin{aligned}
& d_{b c}^{l}=(1-\zeta)\left(d_{c}^{l}+(1-\delta) d_{b}^{u}\right) \\
& d_{b c}^{u}=(1-\zeta)\left(d_{c}^{u}+(1-\delta) d_{b}^{l}\right)
\end{aligned}
$$

where $0 \leq \zeta \leq 1$ is an appropriate chosen dependency coefficient. This will be also assumed in this paper. ${ }^{1}$

Now, to be fully credible, that is, to be sure that (2) is satisfied, the party should prove

$$
D \hat{x}+d_{b c}^{u} \leq 0
$$

This non-equality condition is analogous to (4), with the upper limit $L=0$.

\footnotetext{
${ }^{1}$ Modification of the addition operator has a disadvantage. As far as the usual addition is commutative and associative (i.e. for the intervals $A, B$ and $C$ it holds $A+B=B+A$ and $A+B+C=(A+$ $B)+C=A+(B+C))$, then the modified operator with operations (9) and (10), denoted below as $+_{\zeta}$, is only commutative and not associative, because then $(A+\zeta B)+\zeta \quad \neq A+\zeta(B+\zeta C)$. Thus, practically, the operator $+_{\zeta}$ can be used only for pairs of numbers. But this is actually exactly what is needed in the application considered in this paper.
} 
When a party is compliant with risk $\alpha$, then the part of its distribution that lies above zero is not bigger than $\alpha$, see Fig. 2 for the geometrical interpretation. That is, it holds $D \hat{x}+d_{b c}^{u} \leq 2 \alpha d_{b c}^{u}$. After simple algebraic manipulations this gives the condition

$$
\hat{x}_{c}+\left[1-\left(1+\frac{d_{b c}^{l}}{d_{b c}^{u}}\right) \alpha\right] d_{b c}^{u} \leq(1-\delta) \hat{x}_{b}
$$

This condition is analogous to (6). Thus, to prove the compliance with risk $\alpha$ the party has to fulfill its obligation with the inventory emission estimate increased by the value $\left[1-\left(1+\frac{d_{b c}^{l}}{d_{b c}^{u}}\right) \alpha\right] d_{b c}^{u}$, dependent on its uncertainty.

\subsection{Emission trading}

The above compliance-proving policy can be used to modify the rules of emission trading. The main idea presented in earlier papers (Nahorski et al. 2007; Nahorski and Horabik 2008) involves transferring the emission seller uncertainty to the emission buyer together with the quota of emissions traded and then including it in the buyer's emission balance. Here it is adapted to the asymmetric distributions.

Let us denote by $R_{c}^{u S}=d_{c}^{u S} / \hat{x}_{c}^{S}$ and $R_{c}^{l S}=d_{c}^{l S} / \hat{x}_{c}^{S}$ the respective relative upper and lower spreads of the uncertainty intervals of the seller and by $\hat{E}^{S}$ the amount of estimated emission traded. This emission is associated with lower and upper spreads of the uncertainty intervals $\hat{E}^{S} R_{c}^{l S}$ or $\hat{E}^{S} R_{c}^{u S}$, respectively.

Known limit First, let us consider the simpler case of known limit L. Before the trade the buyer has to satisfy the condition (6), which is reformulated to

$$
\hat{x}_{c}^{B}+d_{c}^{u B}-\left(d_{c}^{l B}+d_{c}^{u B}\right) \alpha \leq L^{B}
$$

After buying $\hat{E}^{S}$ units of emissions from the seller and including the corresponding uncertainty in the formula, the new condition looks like

$$
\hat{x}_{c}^{B}-\hat{E}^{S}+d_{c}^{u B}+\hat{E}^{S} R_{c}^{u S}-\left(d_{c}^{u B}+\hat{E}^{S} R_{c}^{u S}+d_{c}^{l B}+\hat{E}^{S} R_{c}^{l S}\right) \alpha \leq L^{B}
$$

The above conditions differ in the following value, which is called the effective emission (Nahorski et al. 2007)

$$
E_{e f f}=\hat{E}^{S}-\hat{E}^{S} R_{c}^{u S}+\hat{E}^{S}\left(R_{c}^{u S}+R_{c}^{l S}\right) \alpha
$$

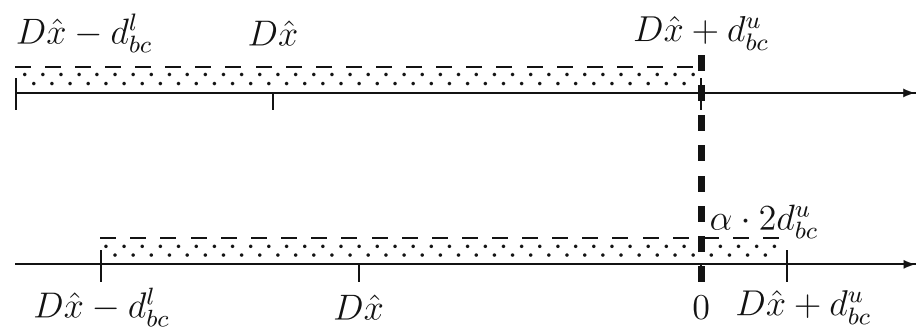

Fig. 2 Full compliance (a) and compliance with risk $\alpha(\mathbf{b})$ in the interval uncertainty approach for the emission reduction case 
which can be transformed to the form

$$
E_{e f f}=\hat{E}^{S}\left\{1-\left[1-\left(1+\frac{d_{c}^{l S}}{d_{c}^{u S}}\right) \alpha\right] R_{c}^{u S}\right\}
$$

The effective emission is smaller than the estimated emission. The bigger the relative upper spread of the uncertainty interval of the seller is, the smaller the effective emission. But it also depends on the ratio $d_{c}^{u S} / d_{c}^{l S}$, and obviously on $\alpha$.

Emission reduction When emission reduction is required, before the trade the buying party checks the following condition

$$
\hat{x}_{c}^{B}+d_{b c}^{u B}-\left(d_{b c}^{u B}+d_{b c}^{l B}\right) \alpha \leq\left(1-\delta^{B}\right) \hat{x}_{b}^{B}
$$

After the transaction the condition changes into

$$
\hat{x}_{c}^{B}-\hat{E}^{S}+d_{b c}^{u B}+\hat{E}^{S} R_{c}^{u S}-\left(d_{b c}^{u B}+\hat{E}^{S} R_{c}^{u S}+d_{b c}^{l B}+\hat{E}^{S} R_{c}^{l S}\right) \alpha \leq\left(1-\delta^{B}\right) \hat{x}_{b}^{B}
$$

Because of partial cancellation of the subtracted estimated emission and its uncertainty in the buyer's emission balance, the effective emission is

$$
E_{e f f}=\hat{E}^{S}\left\{1-\left[1-\left(1+\frac{d_{c}^{l S}}{d_{c}^{u S}}\right) \alpha\right] R_{c}^{u S}\right\}
$$

This is exactly the same formula as (13). The bigger the seller's upper spread of uncertainty interval is, the fewer the purchased units on the account of the buyer. Expressions (13) and (14) reduce emissions estimated with an arbitrary precision to globally comparable values, which can be directly subtracted from a country's estimated emission. This way it is possible to construct a market for the effective emissions, see Nahorski et al. (2007) and Nahorski and Horabik (2007) for details.

\section{Fuzzy uncertainty}

The interval uncertainty approach does not use any information on the distribution of inventory errors. Thus, its results are too conservative. Modeling the uncertainty using the stochastic approach causes problems related to the non-linearities of the underlying algebra. Instead, we propose to use the fuzzy approach in modeling uncertainty distribution. It allows for a good approximation of the distribution while keeping the algebra of the interval calculus simple. A short explanation of fuzzy sets and some related notions is given in the Appendix.

In this paper the fuzzy numbers (see Appendix for a definition) are used to model imperfect knowledge of uncertainty. A fuzzy number is a straight generalization of an ordinary number, whose value is uncertain: the situation that we note pertains to greenhouse gas inventories.

Usually, the main problem with the fuzzy set approach is to determine the membership function. Here, we introduce analytical membership functions dependent on parameters. To estimate the parameters, the function can be fitted to the distribution obtained from Monte Carlo simulations, as shown in the sequel. If there is a lack 
Fig. 3 Membership functions for different values of $\gamma$

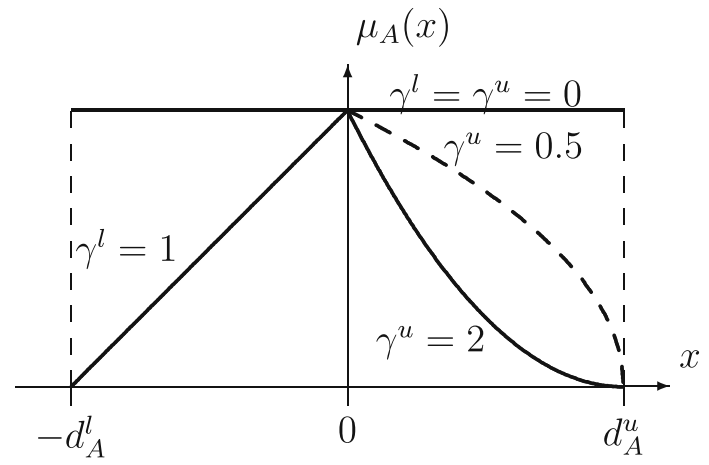

of experimental distributions, the parameter can be fixed to fit the experimenter expectation. $^{2}$

The most popular membership functions are the triangular or trapezoidal ones. These functions are, however, rather inconvenient for our purpose because of their bad approximations of the distribution tails, which are very important in the applications described here.

Consider a family of fuzzy numbers $A^{\gamma}=\left\{\left(x, \mu_{A}^{\gamma}(x)\right) \mid x \in \operatorname{supp} A^{\gamma}\right\}$ indexed by a vector parameter $\gamma=\left[\gamma_{1}^{u}, \gamma_{2}^{l}\right] \in C^{+} \times C^{+}$, with the support supp $A^{\gamma}=\left[-d_{A}^{l}, d_{A}^{u}\right]$. The proposed membership function has the form (see Fig. 3)

$$
\mu_{A}^{\gamma}(x)=\left\{\begin{array}{l}
a\left(1-\frac{x}{d_{A}^{u}}\right)^{\gamma^{u}} \text { for } 0 \leq x \leq d_{A}^{u} \\
a\left(1+\frac{x}{d_{A}^{l}}\right)^{\gamma^{l}} \text { for } d_{A}^{l} \leq x<0
\end{array} \quad \gamma^{l}, \gamma^{u} \neq 0\right.
$$

where $a$ is a normalizing factor used for fitting the membership function to empirical distributions. In the theoretical considerations it can be assumed that the membership function has been normalized and therefore $a=1$ is taken in the sequel. Let us note that taking $\gamma^{l}=\gamma^{u}=0$ we get the even distribution (see Fig. 3) and actually reduce the considerations to the interval case.

Figure 4 presents an estimate of an asymmetric distribution obtained using the Monte Carlo method and presented in Winiwarter and Muik (2007).

\subsection{Compliance}

It is assumed that the uncertainty of the estimate $\hat{x}_{b}$ is described by the membership function

$$
\mu_{\hat{x}_{b}}^{\gamma}(x)=\left\{\begin{array}{l}
\left(1-\frac{x-\hat{x}_{b}}{d_{b}^{u}}\right)^{\gamma_{b}^{u}} \text { for } \hat{x}_{b} \leq x \leq \hat{x}_{b}+d_{b}^{u} \\
\left(1+\frac{x-\hat{x}_{b}}{d_{b}^{l}}\right)^{\gamma_{b}^{l}} \text { for } \hat{x}_{b}-d_{b}^{l} \leq x<\hat{x}_{b}
\end{array}\right.
$$

\footnotetext{
${ }^{2}$ It is perhaps worth mentioning at this point that we treat the fuzzy approach only as an approximation of distribution and algebraic rules for the variables and not to introduce the possibility function, see for example Bandemer (2006), which gives another possible approach to the problem.
} 


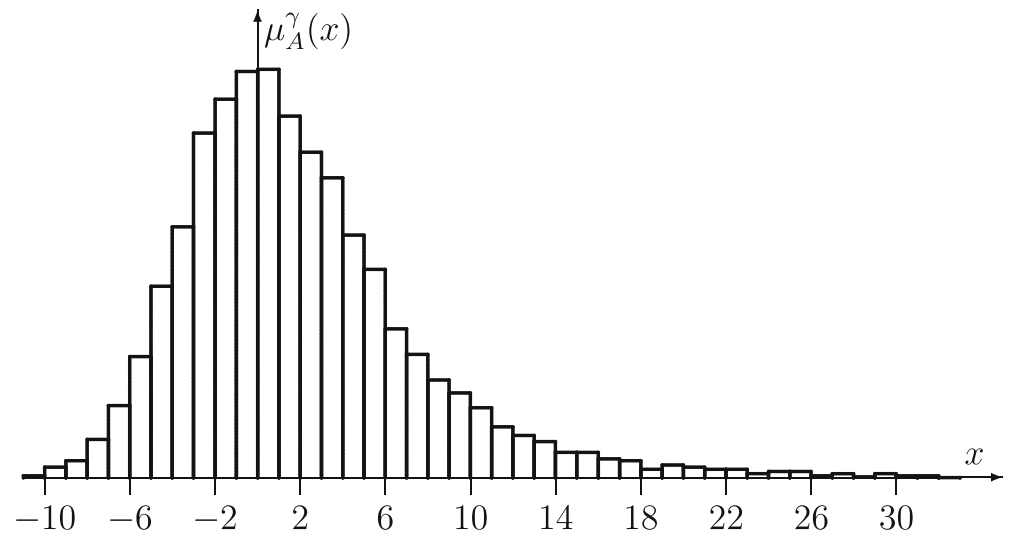

Fig. 4 An estimate of a membership function $\mu_{A}^{\gamma}(x)$ calculated using the Monte Carlo method

and of the estimate $\hat{x}_{c}$ by

$$
\mu_{\hat{x}_{c}}^{\gamma}(x)=\left\{\begin{array}{l}
\left(1-\frac{x-\hat{x}_{c}}{d_{c}^{u}}\right)^{\gamma_{c}^{u}} \text { for } \hat{x}_{c} \leq x \leq \hat{x}_{c}+d_{c}^{u} \\
\left(1+\frac{x-\hat{x}_{c}}{d_{c}^{l}}\right)^{\gamma_{c}^{l}} \text { for } \hat{x}_{c}-d_{c}^{l} \leq x<\hat{x}_{c}
\end{array}\right.
$$

Known limit We start with the exactly known limit case. First, we calculate by integration the whole area $A$ under the membership function. It is the sum of two areas, see Fig. 5

$$
\begin{gathered}
A=A^{l}+A^{u} \\
A^{l}=\int_{\hat{x}_{c}-d_{c}^{l}}^{\hat{x}_{c}}\left(1+\frac{x-\hat{x}_{c}}{d_{c}^{l}}\right)^{\gamma_{c}^{l}} d x=\frac{d_{c}^{l}}{1+\gamma_{c}^{l}} \\
A^{u}=\int_{\hat{x}_{c}}^{\hat{x}_{c}+d_{c}^{u}}\left(1-\frac{x-\hat{x}_{c}}{d_{c}^{u}}\right)^{\gamma_{c}^{u}} d x=\frac{d_{c}^{u}}{1+\gamma_{c}^{u}}
\end{gathered}
$$

We now want to find the distance $x_{c \alpha}$ between $\hat{x}_{c}$ and $\hat{x}_{c}+x_{c \alpha}$, where the latter is the value cutting off the most right $\alpha$ th part of the area under the membership function, see Fig. 5. This area, denoted $A_{\alpha}$, for $0 \leq \alpha \leq A^{u} /\left(A^{l}+A^{u}\right)$, where $A^{l}$ is the area under the left branch of the membership function and $A^{u}$ under the right branch is

$$
A_{\alpha}=\int_{\hat{x}_{c}+x_{c \alpha}}^{\hat{x}_{c}+d_{c}^{u}}\left(1-\frac{x-\hat{x}_{c}}{d_{c}^{u}}\right)^{\gamma_{c}^{u}} d x=\frac{d_{c}^{u}}{1+\gamma_{c}^{u}}\left(1-\frac{x_{c \alpha}}{d_{c}^{u}}\right)^{1+\gamma_{c}^{u}}
$$

Now, it must hold

$$
A_{\alpha}=\alpha A
$$




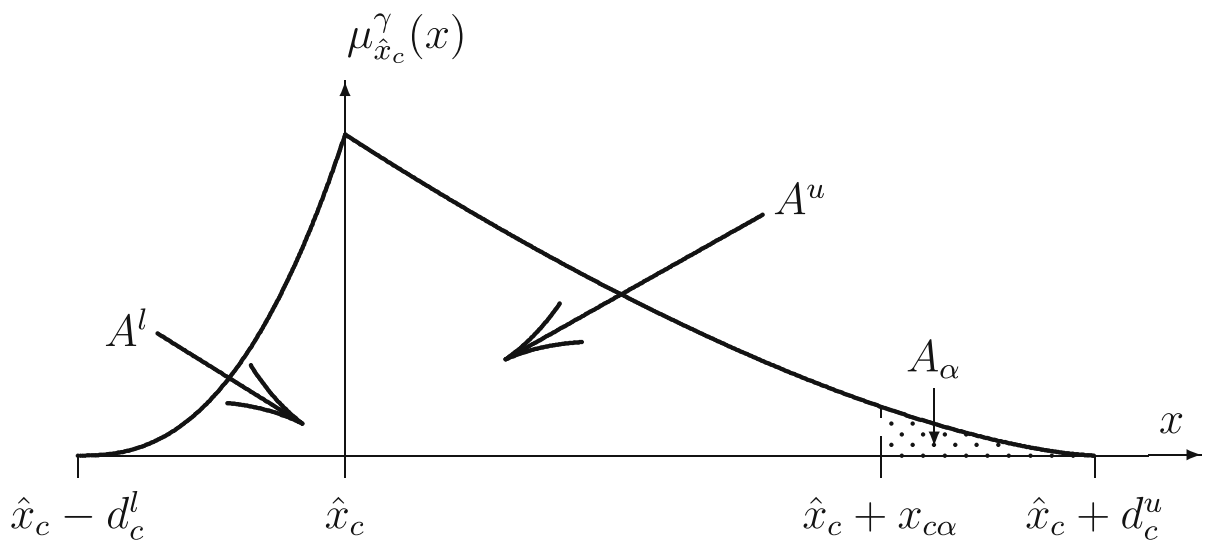

Fig. 5 Definition of areas under asymmetric fuzzy number membership function

which after some algebraic manipulations gives

$$
x_{c \alpha}=\left\{1-\left[\left(1+\frac{d_{c}^{l}}{d_{c}^{u}} \frac{1+\gamma_{c}^{u}}{1+\gamma_{c}^{l}}\right) \alpha\right]^{\frac{1}{1+\gamma_{c}^{u}}}\right\} d_{c}^{u}
$$

Finally, the compliance checking condition is

$$
\hat{x}_{c}+\left\{1-\left[\left(1+\frac{d_{c}^{l}}{d_{c}^{u}} \frac{1+\gamma_{c}^{u}}{1+\gamma_{c}^{l}}\right) \alpha\right]^{\frac{1}{1+\gamma_{c}^{u}}}\right\} d_{c}^{u} \leq L
$$

For the interval uncertainty case $\gamma_{c}^{u}=\gamma_{c}^{l}=0$. Then the above condition is the same as (6). For the symmetric case $d_{c}^{l}=d_{c}^{u}=d_{c}$ and $\gamma_{c}^{l}=\gamma_{c}^{u}=\gamma_{c}$, and the above condition takes the form

$$
\hat{x}_{c}+\left[1-(2 \alpha)^{\frac{1}{1+\gamma_{c}}}\right] d_{c} \leq L
$$

This formula was derived in Nahorski and Horabik (2008).

For the symmetric case, only the range $0 \leq \alpha \leq 0.5$ is practically worth being considered, as for $\alpha=0.5$ the above condition takes the form $\hat{x}_{c} \leq L$, and for $\alpha>0.5$ we would allow for exceeding the limit, that is, for $\hat{x}_{c}>L$. For the asymmetric case the range $0 \leq \alpha \leq A^{u} /\left(A^{l}+A^{u}\right)$ should be considered. Thus, the limiting $\alpha$ can take values greater or smaller than 0.5 . For the interval uncertainty the range will be $0 \leq \alpha \leq d^{u} /\left(d^{l}+d^{u}\right)$.

In addition, let us note that for the right-skewed distributions, as in Fig. 4, the probability of non-compliance is greater than 0.5 when $\hat{x}_{c}$ is equal to the limit $L$. It is a consequence of the fact that in this case of asymmetry, it is more likely that the limit $L$ is exceeded than that it is not attained.

Emission reduction For the emission reduction case, to find the membership function of the fuzzy number $D \hat{x}=\hat{x}_{c}-(1-\delta) \hat{x}_{b}$ as a linear combination of the fuzzy numbers $\hat{x}_{b}$ and $\hat{x}_{c}$, the $\eta$-cuts will be used, see Appendix for an explanation of this 
notion. For the number $\hat{x}_{c}$ the upper $\hat{x}_{c}^{\eta u}$ and the lower $\hat{x}_{c}^{\eta l}$ ends of the $\eta$-cut are as follows, see Fig. 6. For $\hat{x}_{c}^{\eta u}$ we have

$$
\left(1-\frac{\hat{x}_{c}^{\eta u}-\hat{x}_{c}}{d_{c}^{u}}\right)^{\gamma_{c}^{u}}=\eta
$$

Then, assuming $\gamma_{c}^{u} \neq 0$,

$$
\hat{x}_{c}^{\eta u}=\hat{x}_{c}+d_{c}^{u}\left(1-\eta^{\frac{1}{\gamma_{c}^{u}}}\right)
$$

In the same way, for $\hat{x}_{c}^{\eta l}$, assuming $\gamma_{c}^{l} \neq 0$,

$$
\left(1+\frac{\hat{x}_{c}^{\eta l}-\hat{x}_{c}}{d_{c}^{l}}\right)^{\gamma_{c}^{l}}=\eta
$$

and

$$
\hat{x}_{c}^{\eta l}=\hat{x}_{c}-d_{c}^{l}\left(1-\eta^{\frac{1}{\gamma_{c}^{l}}}\right)
$$

For $\gamma_{c}^{u}=0$ or $\gamma_{c}^{l}=0$ we have $\eta=1$. For this case the expression like $\eta^{\frac{1}{\gamma_{c}^{u}}}$ is not formally defined. Thus, we additionally define

$$
\begin{array}{cc}
\eta^{\frac{1}{\gamma_{c}^{u}}}=0, & \text { for } \quad \gamma_{c}^{u}=0 \\
\eta^{\frac{1}{\gamma_{c}^{l}}}=0 & \text { for } \quad \gamma_{c}^{l}=0
\end{array}
$$

The fuzzy number $\hat{x}_{b}$ can be treated analogously. But we consider the number $-(1-\delta) \hat{x}_{b}$. Taking analogous assumptions and additional definitions as above, we now look for $\hat{x}_{b}^{\eta u}$ satisfying

$$
\left(1-\frac{\hat{x}_{b}^{\eta u}+(1-\delta) \hat{x}_{b}}{(1-\delta) d_{b}^{u}}\right)^{\gamma_{b}^{u}}=\eta
$$

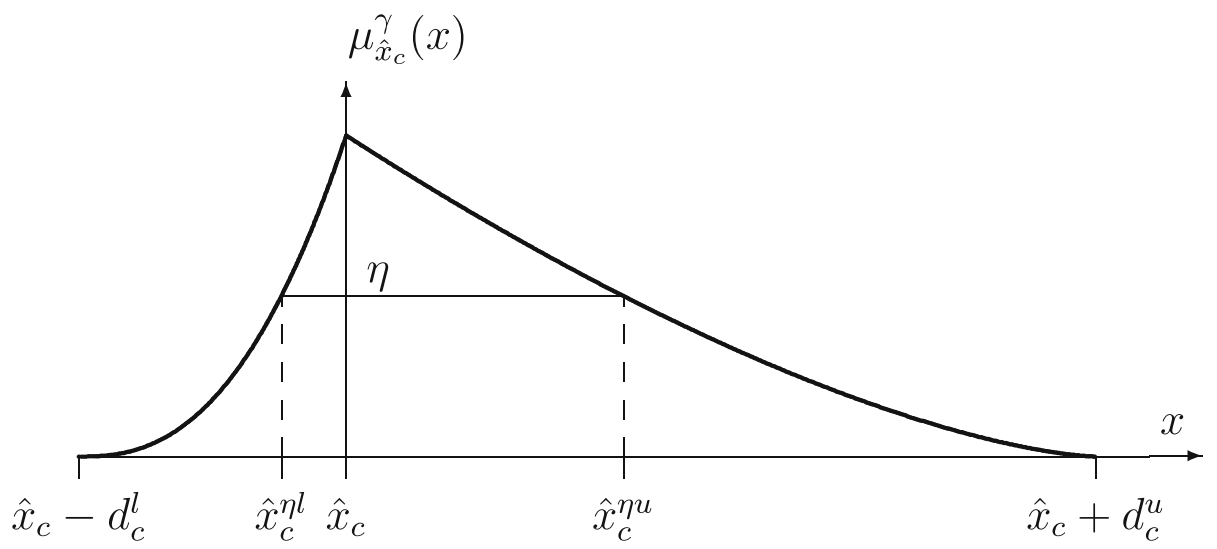

Fig. 6 Asymmetric fuzzy number and definitions of related parameters 
from where the upper end $\hat{x}_{b}^{\eta u}$ of the $\eta$-cut is given by

$$
\hat{x}_{b}^{\eta u}=-(1-\delta) \hat{x}_{b}+d_{b}^{u}(1-\delta)\left(1-\eta^{\frac{1}{\gamma_{b}^{u}}}\right)
$$

For the lower end $\hat{x}_{b}^{\eta l}$ of the $\eta$-cut the equation

$$
\left(1+\frac{\hat{x}_{b}^{\eta l}+(1-\delta) \hat{x}_{b}}{(1-\delta) d_{b}^{l}}\right)^{\gamma_{b}^{l}}=\eta
$$

provides

$$
\hat{x}_{b}^{\eta l}=-(1-\delta) \hat{x}_{b}-d_{b}^{l}(1-\delta)\left(1-\eta^{\frac{1}{\gamma_{b}^{l}}}\right)
$$

Finally, the $\eta$-cut of the number $D \hat{x}$ is obtained by applying the modified interval calculus rules (9) and (10) for the sum of the $\eta$-cuts of the numbers $\hat{x}_{c}$ and $-(1-\delta) \hat{x}_{b}$. Thus

$$
\begin{aligned}
& D \hat{x}^{\eta u}=D \hat{x}+(1-\zeta)\left[d_{c}^{u}\left(1-\eta^{\frac{1}{\gamma_{c}^{u}}}\right)+d_{b}^{l}(1-\delta)\left(1-\eta^{\frac{1}{\gamma_{b}^{l}}}\right)\right] \\
& D \hat{x}^{\eta l}=D \hat{x}-(1-\zeta)\left[d_{c}^{l}\left(1-\eta^{\frac{1}{\gamma_{c}^{l}}}\right)+d_{b}^{u}(1-\delta)\left(1-\eta^{\frac{1}{\gamma_{b}^{u}}}\right)\right]
\end{aligned}
$$

The above equations show dependences of $D \hat{x}^{\eta l}$ and $D \hat{x}^{\eta u}$ on $\eta$, that is, they are the reverse functions of the two branches of the membership function $\mu_{D \hat{x}}^{\gamma}(x)$, see Fig. 6 .

Let us now transform (18) to

$$
1-\frac{D \hat{x}^{\eta u}-D \hat{x}}{d_{b c}^{u}}=\frac{d_{c}^{u} \eta^{1 / \gamma_{c}^{u}}+d_{b}^{l}(1-\delta) \eta^{1 / \gamma_{b}^{l}}}{d_{c}^{u}+d_{b}^{l}(1-\delta)}
$$

where $d_{b c}^{u}$ is given by (10), and define $\gamma_{b c}^{u}$ to satisfy the equation

$$
\frac{d_{c}^{u} \eta^{1 / \gamma_{c}^{u}}+d_{b}^{l}(1-\delta) \eta^{1 / \gamma_{b}^{l}}}{d_{c}^{u}+d_{b}^{l}(1-\delta)}=\eta^{\frac{1}{\gamma_{b c}^{u}}}
$$

From the above

$$
\gamma_{b c}^{u}=\frac{1}{\log _{\eta} \frac{d_{c}^{u} \eta^{1 / \gamma_{c}^{u}}+d_{b}^{l}(1-\delta) \eta^{1 / \gamma_{b}^{l}}}{d_{c}^{u}+d_{b}^{l}(1-\delta)}}=\frac{\log \eta}{\log \frac{d_{c}^{u} \eta^{1 / \gamma_{c}^{u}}+d_{b}^{l}(1-\delta) \eta^{1 / \gamma_{b}^{l}}}{d_{c}^{u}+d_{b}^{l}(1-\delta)}}
$$

In the spirit of earlier additional definitions we also define

$$
\gamma_{b c}^{u}=0 \quad \text { for } \quad \gamma_{c}^{u}=0 \text { or } \quad \gamma_{b}^{l}=0
$$

Now it is possible to write the right branch of the membership function as

$$
\mu_{\hat{x}_{b c}}^{\gamma u}(x)=\left(1-\frac{x-D \hat{x}}{d_{b c}^{u}}\right)^{\gamma_{b c}^{u}} \quad D \hat{x} \leq x \leq D \hat{x}+d_{b c}^{u}
$$


Likewise we get

$$
\mu_{\hat{x}_{b c}}^{\gamma l}(x)=\left(1+\frac{x-D \hat{x}}{d_{b c}^{l}}\right)^{\gamma_{b c}^{l}} \quad D \hat{x}-d_{b c}^{l} \leq x \leq D \hat{x}
$$

where $d_{b c}^{l}$ is given by (9), and

$$
\gamma_{b c}^{l}=\frac{1}{\log _{\eta} \frac{d_{c}^{l} \eta^{1 / \gamma_{c}^{l}}+d_{b}^{u}(1-\delta) \eta^{1 / \gamma_{b}^{u}}}{d_{c}^{l}+d_{b}^{u}(1-\delta)}}=\frac{\log \eta}{\log \frac{d_{c}^{l} \eta^{1 / \gamma_{c}^{l}}+d_{b}^{u}(1-\delta) \eta^{1 / \gamma_{b}^{u}}}{d_{c}^{l}+d_{b}^{u}(1-\delta)}}
$$

with

$$
\gamma_{b c}^{l}=0 \quad \text { for } \quad \gamma_{c}^{l}=0 \text { or } \quad \gamma_{b}^{u}=0
$$

Now, the most right $\alpha$ th part of the area under the membership function (22) is

$$
A_{\alpha}=\int_{D \hat{x}+x_{\alpha}}^{D \hat{x}+d_{b c}^{u}}\left(1-\frac{x-D \hat{x}}{d_{b c}^{u}}\right)^{\gamma_{b c}^{u}} d x=\frac{d_{b c}^{u}}{1+\gamma_{b c}^{u}}\left(1-\frac{x_{\alpha}}{d_{b c}^{u}}\right)^{1+\gamma_{b c}^{u}}
$$

and the area under the entire membership function (22)-(23) is

$$
\begin{aligned}
A & =\int_{D \hat{x}-d_{b c}^{l}}^{D \hat{x}}\left(1+\frac{x-D \hat{x}}{d_{b c}^{l}}\right)^{\gamma_{b c}^{l}} d x+\int_{D \hat{x}}^{D \hat{x}+d_{b c}^{u}}\left(1-\frac{x-D \hat{x}}{d_{b c}^{u}}\right)^{\gamma_{b c}^{u}} d x= \\
& =\frac{d_{b c}^{l}}{1+\gamma_{b c}^{l}}+\frac{d_{b c}^{u}}{1+\gamma_{b c}^{u}}
\end{aligned}
$$

As $A_{\alpha}=\alpha A$, its solution for $x_{\alpha}$, denoted $x_{b c \alpha}$, has the form

$$
x_{b c \alpha}=\left\{1-\left[\left(1+\frac{d_{b c}^{l}}{d_{b c}^{u}} \frac{1+\gamma_{b c}^{u}}{1+\gamma_{b c}^{l}}\right) \alpha\right]^{\frac{1}{1+\gamma_{b c}^{u}}}\right\} d_{b c}^{u}
$$

and finally the compliance condition is

$$
\hat{x}_{c}+\left\{1-\left[\left(1+\frac{d_{b c}^{l}}{d_{b c}^{u}} \frac{1+\gamma_{b c}^{u}}{1+\gamma_{b c}^{l}}\right) \alpha\right]^{\frac{1}{1+\gamma_{b c}^{u}}}\right\} d_{b c}^{u} \leq(1-\delta) \hat{x}_{b}
$$

This condition is analogous to (17). For the interval case $\gamma_{b c}^{l}=\gamma_{b c}^{u}=0$ and (27) reduces to (12). For the symmetric distribution $d_{b c}^{l}=d_{b c}^{u}=d_{b c}$ and $\gamma_{b c}^{l}=\gamma_{b c}^{u}=\gamma_{b c}$ and it reduces to

$$
\hat{x}_{c}+\left[1-(2 \alpha)^{\frac{1}{1+\gamma_{b c}}}\right] d_{b c} \leq(1-\delta) \hat{x}_{b}
$$

The condition (28) has been derived in Nahorski and Horabik (2007). 


\subsection{Emission trading}

The formula for the effective emission can be quite easily obtained for the symmetric distribution (28) using derivations similar to the interval case. Before the trade, the buying party has to satisfy the condition

$$
\hat{x}_{c}^{B}+\left[1-(2 \alpha)^{\frac{1}{1+\gamma_{b c}^{B}}}\right] d_{b c}^{B} \leq\left(1-\delta^{B}\right) \hat{x}_{b}^{B}
$$

and after buying $\hat{E}^{S}$ emission units from the seller it becomes

$$
\hat{x}_{c}^{B}-\hat{E}^{S}+\left[1-(2 \alpha)^{\frac{1}{1+\gamma_{b c}^{B}}}\right]\left(d_{b c}^{B}+\hat{E}^{S} R_{c}^{S}\right) \leq\left(1-\delta^{B}\right) \hat{x}_{b}^{B}
$$

Then the effective emission is

$$
E_{e f f}=\hat{E}^{S}\left\{1-\left[1-(2 \alpha)^{\frac{1}{1+\gamma_{b c}^{B}}}\right] R_{c}^{S}\right\}
$$

where $R_{c}^{S}=d_{c}^{S} / \hat{x}_{c}^{S}$ and for the symmetric distributions $d_{c}^{S}=d_{c}^{u S}=d_{c}^{l S}$.

However, the problem becomes more difficult for the asymmetric distributions, as then the uncertainty distribution bounds $d_{b c}^{l}$ and $d_{b c}^{u}$ enter non-linearly into the compliance condition (27). This is why linearization is now used to obtain the result. The exact derivation is presented in Electronic Supplementary Material. That way the following expression for the effective emission is obtained

$$
E_{e f f}=\hat{E}^{S}\left\{1-\left\{1-\left[\left(1+\frac{d_{c}^{l S}}{d_{c}^{u S}}\right) \alpha\right]^{\frac{1}{1+\gamma_{b c}^{u B}}}\right\} R_{c}^{u S}\right\}
$$

It generalizes expressions for simpler cases. In particular, for the known limit case the following substitution should be made: $\gamma_{b c}^{u B} \rightarrow \gamma_{c}^{u B}$. For the symmetric distributions the substitutions are: $d_{c}^{l S} \rightarrow d^{S}, d_{c}^{u S} \rightarrow d^{S}, \gamma_{b c}^{u B} \rightarrow \gamma_{b c}^{B}$, which provide (29). For the interval uncertainty: $\gamma_{b c}^{u B} \rightarrow 0$, which gives (14).

In comparison with the formula (14) for the interval uncertainty, the formulas (29) and (30) depend on parameters $\gamma_{b c}^{B}$ or $\gamma_{b c}^{u B}$ of the emission buyer uncertainty distributions. This would considerably complicate the market, as the traded quota depends in such a case both on the seller and the buyer uncertainty distributions. This problem will not be discussed in this paper.

\subsection{Equivalence of approaches}

We start here with a summary of results. Table 1 provides relevant formulas for compliance condition and effective emission in the case of known limit for various types of uncertainty models considered.

Let us note that, for a given case, the same compliance condition or the same effective emissions can be obtained for the interval model as for the fuzzy model, choosing an appropriate value of $\alpha$ in the former one. Let us denote by $\alpha_{I}$ the value for the interval model and by $\alpha_{F}$ for the fuzzy one. It can be noticed that actually it is enough to consider only the asymmetric cases, as the results for the symmetric cases are obtained taking specific values od parameters. 
Table 1 Model review

\begin{tabular}{lll}
\hline Model & Compliance checking & Effective emission \\
\hline $\begin{array}{l}\text { Interval } \\
\text { symmetric }\end{array}$ & $\hat{x}_{c}+(1-2 \alpha) d_{c} \leq L$ & $E_{\text {eff }}=\hat{E}^{s}\left[1-(1-2 \alpha) R_{c}^{S}\right]$ \\
$\begin{array}{l}\text { Interval } \\
\text { asymmetric }\end{array}$ & $\hat{x}_{c}+\left[1-\left(1+\frac{d_{c}^{l}}{d_{c}^{u}}\right) \alpha\right] d_{c}^{u} \leq L$ & $E_{\text {eff }}=\hat{E}^{s}\left\{1-\left[1-\left(1+\frac{d_{c}^{l S}}{d_{c}^{u S}}\right) \alpha\right] R_{c}^{u S}\right\}$ \\
Fuzzy & $\hat{x}_{c}+\left[1-(2 \alpha)^{\frac{1}{1+\gamma_{c}}}\right] d_{c} \leq L$ & $E_{e f f}=\hat{E}^{s}\left\{1-\left[1-(2 \alpha)^{\frac{1}{1+\gamma_{c}^{B}}}\right] R_{c}^{S}\right\}$ \\
$\quad \begin{array}{l}\text { symmetric } \\
\text { Fuzzy }\end{array}$ & $\hat{x}_{c}+\{1-[(1+$ & $E_{e f f}=\hat{E}^{s} \times$ \\
$\quad$ asymmetric & $\left.\left.\left.\frac{d_{c}^{l}}{d_{c}^{u}} \frac{1+\gamma_{c}^{u}}{1+\gamma_{c}^{l}}\right) \alpha\right]^{\frac{1}{1+\gamma_{c}^{u}}}\right\} d_{c}^{u} \leq L$ & $\left\{1-\left\{1-\left[\left(1+\frac{d_{c}^{l S}}{d_{c}^{u S}}\right) \alpha\right]^{\frac{1}{1+\gamma_{c}^{u B}}}\right\} R_{c}^{u S}\right\}$ \\
\hline
\end{tabular}

Equaling the effective emissions $E_{e f f, F}=E_{e f f, I}$, from the second and the fourth rows in the last column of the Table 1 , after simple algebraic manipulations we arrive at the following condition

$$
\left[\left(1+\frac{d_{c}^{l S}}{d_{c}^{u S}}\right) \alpha_{I}\right]^{1+\gamma_{b c}^{u B}}=\left(1+\frac{d_{c}^{l S}}{d_{c}^{u S}}\right) \alpha_{F}
$$

If the cases $\alpha_{F}=0$ (no noncompliance risk) and $\gamma_{b c}^{u B}=0$ (interval uncertainty) are excluded, then

$$
\frac{\alpha_{I}}{\alpha_{F}}=\left[\left(1+\frac{d_{c}^{l S}}{d_{c}^{u S}}\right) \alpha_{I}\right]^{-\gamma_{b c}^{u B}}
$$

Thus we have

$$
\alpha_{I}>\alpha_{F} \quad \text { for } \quad \alpha_{I} \leq \frac{d_{c}^{u S}}{d_{c}^{u S}+d_{c}^{l S}} \quad \text { and } \quad \gamma_{b c}^{u B}>0
$$

Both conditions are very mild. The second is obviously satisfied. Taking into account that in the up to now considered cases $d_{c}^{u S} \geq d_{c}^{l S}$, then in the first condition the upper limit is not smaller than 0.5 , which is true for the symmetric case.

For the compliance checking, comparing formulas from the second and the fourth rows in the middle column we get

$$
\left[\left(1+\frac{d_{c}^{l}}{d_{c}^{u}}\right) \alpha_{I}\right]^{1+\gamma_{c}^{u}}=\left(1+\frac{d_{c}^{l}}{d_{c}^{u}} \frac{1+\gamma_{c}^{u}}{1+\gamma_{c}^{l}}\right) \alpha_{F}
$$

from where

$$
\frac{\alpha_{I}}{\alpha_{F}}=\frac{1+\frac{d_{c}^{l}}{d_{c}^{u}} \frac{1+\gamma_{c}^{u}}{1+\gamma_{c}^{l}}}{1+\frac{d_{c}^{l}}{d_{c}^{u}}}\left[\left(1+\frac{d_{c}^{l}}{d_{c}^{u}}\right) \alpha_{I}\right]^{-\gamma_{c}^{u}}
$$

Now, if $\alpha_{I} \leq \frac{d_{c}^{u}}{d_{c}^{u}+d_{c}^{l}}$ and $\gamma_{c}^{u} \geq \gamma_{c}^{l}>0$, and at least one of these conditions is strict, then again

$$
\alpha_{I}>\alpha_{F}
$$


Table 2 Dependence of $\alpha_{I}$ on $\alpha_{F}$ and $\gamma_{b c}^{u B}$

\begin{tabular}{ccccccc}
\hline$\alpha_{F} \downarrow \gamma_{b c}^{u B} \rightarrow$ & 0.1 & 0.5 & 1 & 1.5 & 2 & 2.5 \\
\hline$d_{c}^{l} / d_{c}^{u}=0.2$ & & & & & & \\
0.05 & 0.06 & 0.13 & 0.20 & 0.27 & 0.33 & 0.37 \\
0.10 & 0.12 & 0.20 & 0.29 & 0.36 & 0.41 & 0.45 \\
0.15 & 0.18 & 0.27 & 0.35 & 0.42 & 0.47 & 0.51 \\
0.20 & 0.23 & 0.32 & 0.41 & 0.47 & 0.52 & 0.55 \\
0.25 & 0.28 & 0.37 & 0.46 & 0.51 & 0.56 & 0.59 \\
$d_{c}^{l} / d_{c}^{u}=0.5$ & & & & & & \\
0.05 & 0.06 & 0.12 & 0.18 & 0.24 & 0.28 & 0.32 \\
0.10 & 0.12 & 0.19 & 0.26 & 0.31 & 0.35 & 0.39 \\
0.15 & 0.17 & 0.25 & 0.32 & 0.37 & 0.41 & 0.44 \\
0.20 & 0.22 & 0.30 & 0.37 & 0.41 & 0.45 & 0.47 \\
0.25 & 0.27 & 0.35 & 0.41 & 0.45 & 0.48 & 0.50 \\
$d_{c}^{l} / d_{c}^{u}=1$ (symmetric case) $)$ & & & & \\
0.05 & 0.06 & 0.11 & 0.16 & 0.20 & 0.23 & 0.26 \\
0.10 & 0.12 & 0.17 & 0.22 & 0.26 & 0.29 & 0.32 \\
0.15 & 0.17 & 0.22 & 0.27 & 0.31 & 0.33 & 0.35 \\
0.20 & 0.22 & 0.27 & 0.32 & 0.35 & 0.37 & 0.38 \\
0.25 & 0.27 & 0.32 & 0.35 & 0.38 & 0.40 & 0.41 \\
\hline & & & & & &
\end{tabular}

Thus, the noncompliance risk parameter $\alpha$ in the interval uncertainty model has to be greater than in the fuzzy model to get the same compliance conditions or effective emissions.

Dependence of $\alpha_{I}$ on $\alpha_{F}$ and $\gamma_{b c}^{u B}$ for effective emissions is shown in Table 2. The results show that $\alpha_{I}$ rises quickly when $\gamma_{b c}^{u B}$ rises. In cases considered in our calculations, estimates of $\gamma_{b c}^{u B}$ close to or much higher than 1.5 were obtained. Then, practically it seems that $\alpha_{I} \geq 0.3$ should be taken even for small values of $\alpha_{F}$.

An interpretation of these results is quite straightforward. Within the considered family of distributions, ignorance of the uncertainty distribution in the interval case requires a greater reduction. To obtain the same effective emissions as for the fuzzy uncertainties, a bigger substitutional non-compliance risk should be adopted in the interval approach. Thus, for $\alpha_{I}$, at least the values 0.3 or higher should be taken

Fig. 7 Fit of a membership function $\mu_{A}^{\gamma}(x)$ to the histogram for emission of $\mathrm{CO}_{2}$

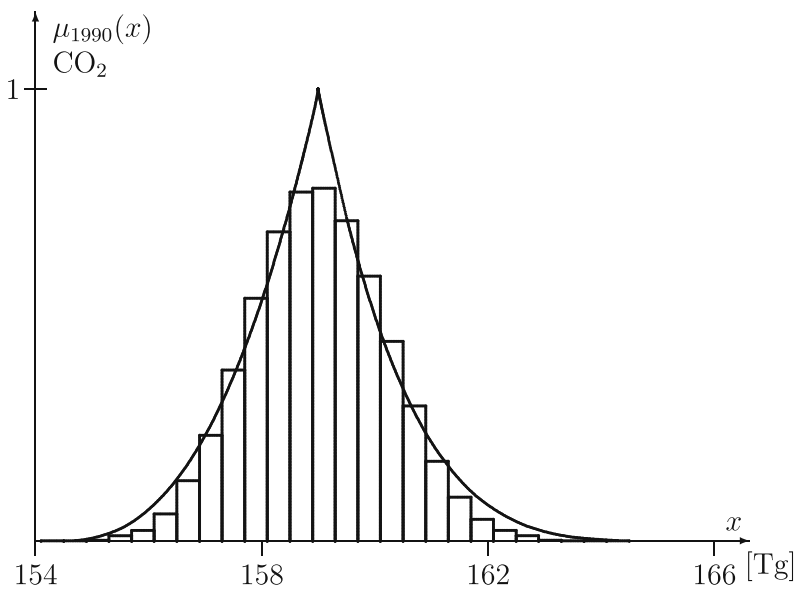


Fig. 8 Fit of a membership function $\mu_{A}^{\gamma}(x)$ to the histogram for emission of $\mathrm{CH}_{4}$

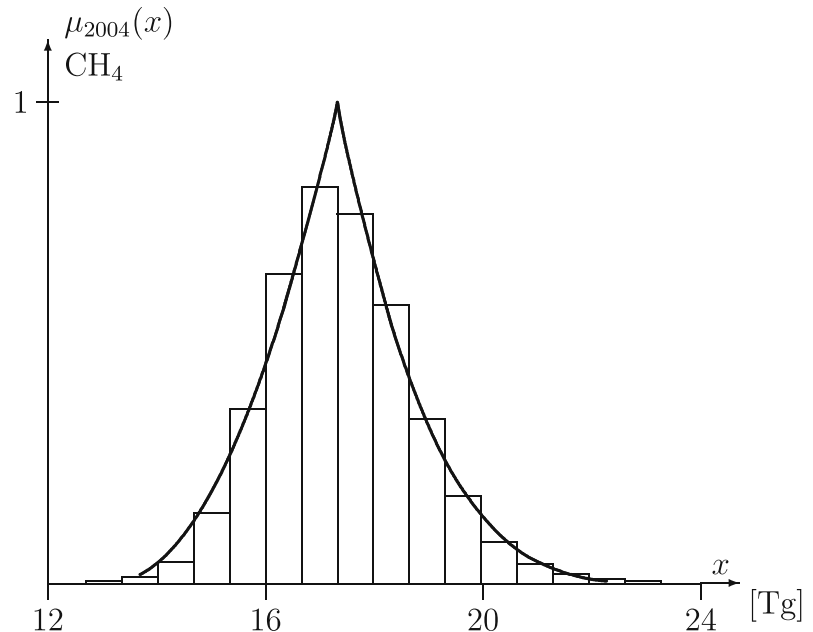

to compensate for ignorance of the exact knowledge of the uncertainty interval distribution, even if a small non-compliance risk is actually meant.

\section{An example}

In the example the data from the Monto Carlo simulation presented in Ramirez et al. (2006) are used. Uncertainty distributions of emissions of three gases, carbon dioxide $\left(\mathrm{CO}_{2}\right)$, methane $\left(\mathrm{CH}_{4}\right)$, and fluorine $(\mathrm{F})$, are considered. The uncertainty distributions were chosen to illustrate the proposed rules of trade and are depicted in Figs. 7, 8 and 9 together with fits of the distribution functions (15). It is assumed that each emission is related to different companies, called $\mathrm{CO}_{2}, \mathrm{CH}_{4}$ and $\mathrm{F}$, respectively. Table 3 contains parameters of the distributions obtained from the fits.

Fig. 9 Fit of a membership function $\mu_{A}^{\gamma}(x)$ to the histogram for emission of $\mathrm{F}$

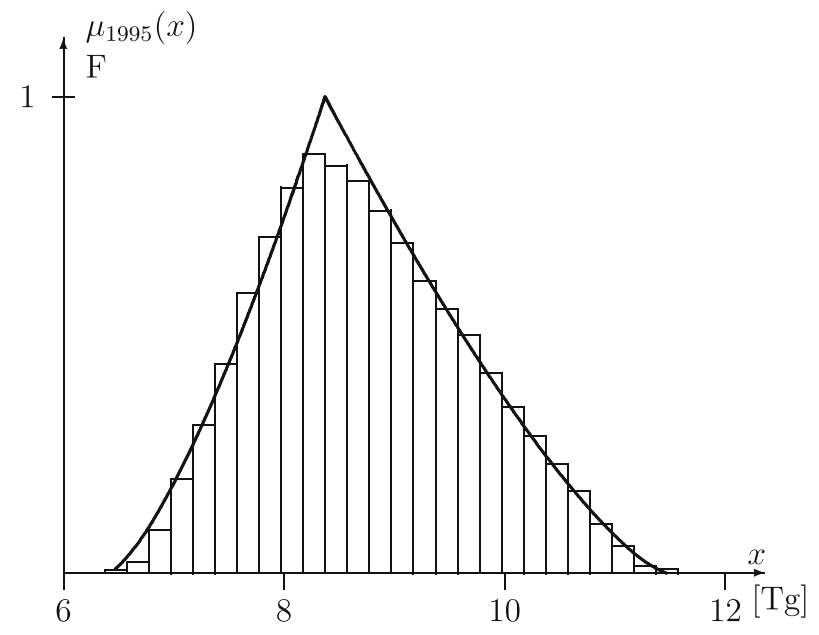


Table 3 Parameters of the distributions

\begin{tabular}{lllll}
\hline Distribution & $d^{l}[\mathrm{Tg}]$ & $\gamma^{l}$ & $\gamma^{u}$ & $d^{u}[\mathrm{Tg}]$ \\
\hline $\mathrm{CO}_{2}$ & 4.8 & 2.6 & 4.5 & 6.9 \\
$\mathrm{CH}_{4}$ & 4.3 & 2.1 & 3.9 & 6.7 \\
$\mathrm{~F}$ & 2.0 & 1.4 & 1.4 & 3.1 \\
\hline
\end{tabular}

We do not consider the compliance, only the trade. Let us then suppose that the three companies mentioned: $\mathrm{CO}_{2}, \mathrm{CH}_{4}$, and $\mathrm{F}$, want to trade with each other. The uncertainty of emissions in company $\mathrm{CO}_{2}$ is small, less than 4 percent, while in the rest it is around 38 percent. On the other hand, the shape of the uncertainty distributions of $\mathrm{CO}_{2}$ and $\mathrm{CH}_{4}$ are similar, with values $\gamma$ of the order of 2-2.5 for the lower and 4-4.5 for the upper branch, while the shape of $\mathrm{F}$ is close to triangular, with $\gamma$ equal to 1.4. In Table 4 the values of $E_{e f f}$ are depicted for three assumed trades, when each company in turn is the seller while the others are buyers. Two values of the original non-compliance risk $\alpha=0.05$ or 0.1 were assumed and substitutional values of $\alpha_{I}$ are given in the right-hand side of the table. Most of them are of the order of 0.4 . For $\mathrm{CO}_{2}$, with small uncertainty, the values $E_{\text {eff }}^{1}$ are only slightly smaller than 1. The values $E_{\text {eff }}^{2}$ and $E_{\text {eff }}^{3}$ are much smaller, around 0.8-0.9.

Let us note that for the fuzzy distribution there is no unique substitutional risk parameter $\alpha_{I}$ related with the seller, because it also depends on who the buyer is. This is what causes problems in the trade as compared to the interval case. A way of avoiding this might be that a common value 0.4 or a smaller one, like 0.35 , is taken for $\alpha_{I}$ to organize the market with a substitutional interval uncertainty. This way the market scheduled in Nahorski et al. (2007) can be applied. A market with substitutional risk parameters $\alpha_{I}$ dependent on the buyer is, however, an interesting question. It will be considered elsewhere.

\section{Conclusions}

The paper deals with the problem of checking compliance of pollutant emissions with a given limit in the case where the observed emission values are highly uncertain with asymmetric uncertainty distributions. High uncertainty should be also considered in trading in emission permits, which is frequently used to minimize the emission abatement cost, and this is also done in the paper. Asymmetric uncertainty is evidenced by recent investigations, and particularly by Monte Carlo simulations of uncertainty distributions.

Table 4 Effective emissions in the trade and substitutional values of $\alpha_{I}$ for interval uncertainty

\begin{tabular}{llllllll}
\hline Emission & $R_{c}^{u}$ & $E_{\text {eff }}^{1}$ & $E_{\text {eff }}^{2}$ & $E_{\text {eff }}^{3}$ & $\alpha_{I}^{1}$ & $\alpha_{I}^{2}$ & $\alpha_{I}^{3}$ \\
\hline$\alpha=0.05$ & & & & & & & \\
$\mathrm{CO}_{2}$ & 0.043 & Seller & 0.86 & 0.86 & - & 0.21 & 0.36 \\
$\mathrm{CH}_{4}$ & 0.385 & 0.98 & Seller & 0.85 & 0.39 & - & 0.36 \\
$\mathrm{~F}$ & 0.371 & 0.97 & 0.75 & Seller & 0.39 & 0.37 & - \\
$\alpha=0.1$ & & & & & & & \\
$\mathrm{CO}_{2}$ & 0.043 & Seller & 0.89 & 0.90 & - & 0.28 & 0.41 \\
$\mathrm{CH}_{4}$ & 0.385 & 0.99 & Seller & 0.88 & 0.44 & - & 0.42 \\
$\mathrm{~F}$ & 0.371 & 0.98 & 0.79 & Seller & 0.44 & 0.42 & - \\
\hline
\end{tabular}


Asymmetry of distributions biases the compliance and trading results, and it constitutes an additional issue in troubles related to uncertainty of emission inventories. This is due to unequal probabilities of occurrence of the real emission below and above the nominal inventory value. The Monte Carlo simulations of national greenhouse gases inventories (Winiwarter and Muik 2007; Ramirez et al. 2006) show that the distributions are right-skewed, that is, real emissions higher that the nominal value are more likely than the smaller ones. This means that even if the nominal inventory value is exactly equal to the given limit and is considered to be compliant according to the present standard, it is actually more probable that the real emission is non-compliant than that it is compliant.

An interesting case ${ }^{3}$ of an asymmetric distribution of uncertainty is connected with the risk of valuing forest carbon offsets caused by accidental losses, for example, due to wildfires (Hurteau et al. 2009). The uncertainty there has a specific one-sided distribution. This case has already entered the implementation stage in the United States forest carbon storage project (Mignone et al. 2009). However, the solutions applied there take into account that the related uncertainty is eventually resolved in the future, as the damages are known after they have happened. This is in contrast with the case discussed in this paper, where uncertainties are an inherent part of data considered at all stages of decision making.

The idea proposed in this paper is based on grounding the derivations in the fuzzy set approach. A family of fuzzy numbers depending on free parameters is introduced. These parameters can be chosen to appropriately shape the distribution of uncertainty. The approach provides the closed form formulas, which can be used for designing a market for effective emission permits. A market with the effective emission permits has been outlined in earlier papers (Nahorski et al. 2007; Nahorski and Horabik 2008) for the symmetric case. That construction is also valid in the asymmetric case discussed in this paper, after appropriate adaptation. However, for the most general case of asymmetric membership functions, a closed analytical solution could not be found. An approximate solution was considered for this case, and a generalized rule for compliance has been derived.

Application of the fuzzy numbers and consideration of asymmetric distributions enabled us to much more precisely determine the required level of reduced inventories to obtain a high likelihood of fulfilling the given limit or reduction. Moreover, better accuracy in terms of determining the level give rise to better scaling of the amounts of emission emitted by parties for use in trading, which has a measurable financial meaning. Approximating distribution by a function dependent on parameters allowed us to derive the analytical expressions for reduction of emissions and for scaling the traded emissions. The distribution parameters have been acquired by fitting the distribution functions to the data from the Monte Carlo simulations.

The results obtained are generalizations of the results derived for the interval and symmetric uncertainty models. However, it was shown that the rules for the interval case can be used instead of the generalized ones, provided that the appropriately higher value of the risk of non-compliance is substituted in the interval case.

Although the fits of the functions presented in this paper to the data are quite good, except perhaps in the central part of the uncertainty interval, the question of

${ }^{3}$ This direction of research has been brought to our attention by one of undisclosed reviewers. 
a possibly better fit to the data has been raised by one of the anonymous reviewers. As this is certainly possible with a more flexible class of functions, the possibility of obtaining a close analytical solution may be challenging. It will be a subject of further investigations.

Open Access This article is distributed under the terms of the Creative Commons Attribution Noncommercial License which permits any noncommercial use, distribution, and reproduction in any medium, provided the original author(s) and source are credited.

\section{Appendix: Fuzzy sets and fuzzy numbers}

To introduce the notion of a fuzzy set, let us first consider a classical set $A$ from an universe $U$. This can be conveniently described by the characteristic function $\chi_{A}$ defined as

$$
\chi_{A}(u)=\left\{\begin{array}{l}
1 \text { if } u \in A \\
0 \text { if } u \notin A
\end{array}\right.
$$

which says that a point $u \in U$ belongs to the set, if $\chi_{A}(u)=1$, or does not belong, if $\chi_{A}(u)=0$.

In a fuzzy set the characteristic function $\chi_{A}$ is generalized to take any value from the interval $[0,1]$. It is then called a membership function and is denoted $\mu_{A}$. The value of a membership function $\mu_{A}(u)$ reflects the degree of acceptance of the point $u$ to the set. Thus, a fuzzy set is characterized by the set $A$ and the membership function $\mu_{A}$. A usual set is then a special fuzzy set with the membership function being the characteristic function. A comparison of a membership function and a characteristic function of a set is shown in Fig. 10.

A fuzzy set can be also fully characterized by a family of so-called $\eta$-cuts ${ }^{4}$ denoted by $A_{\eta}$, that is, points of $U$, for which the value $\mu_{A}(u)$ assumes at least the value $\eta$. See Fig. 10, where an example of a $\eta$-cut for $\eta=0.5$ is depicted.

Two additional notions connected with a fuzzy set are worth mentioning. One is the support, called supp $A$, which is the set of points $u$, for which the membership function is positive, that is,

$$
\operatorname{supp} A=\left\{u \in U: \mu_{A}(u)>0\right\}
$$

Another definition of the support may be formulated using $\eta$-cuts, as

$$
\text { supp } A=\lim _{\eta \rightarrow 0} A_{\eta}
$$

The second notion is the core of the fuzzy set, called core $A$, which is the set of points, for which the membership function is equal 1, that is,

$$
\text { core } A=\left\{u \in U: \mu_{A}(u)=1\right\}
$$

Using the notion of the $\eta$-cuts we may also write

$$
\text { core } A=A_{1}
$$

\footnotetext{
${ }^{4}$ Here we name the $\eta$-cut of a fuzzy set $A$ the notion usually called the $\alpha$-cut, i.e. the set $A_{\eta}=\{x \in$ $\left.\operatorname{supp} A \mid \mu_{A}(x) \geq \eta\right\}$, for $\eta \in(0,1]$. 
Fig. 10 The characteristic function and a membership functions of the set $A$

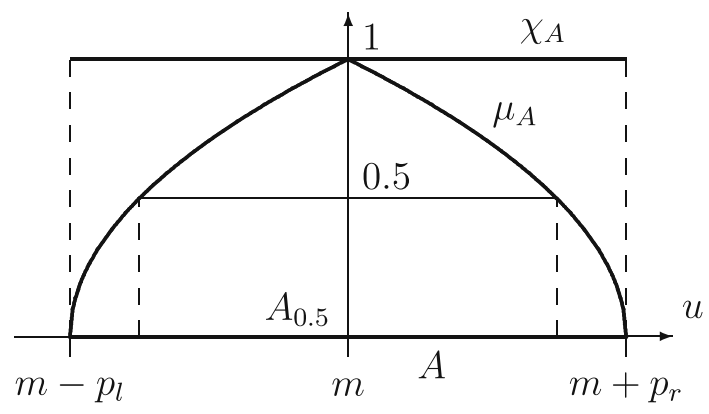

A fuzzy set $A$ is called a fuzzy number, if it satisfies three additional conditions:

1. core $A$ consists of only one point.

2. The membership function does not increase starting from the core point toward both sides.

3. Every $\eta$-cut is a (connected) closed interval.

A weaker definition of a fuzzy number is often used, with the first condition replaced by

1' There is a point belonging to the core $A$.

But in this paper we use the former stronger definition.

The $\eta$-cuts for a fuzzy number form a family of intervals. Each interval can be interpreted as our measure of knowledge of the core value. Values of the level $\eta$ close to 1 mean that we are highly convinced that the core value is precise. Small values of $\eta$, close to 0 , mean that our conviction is low. See also Dubois and Prade (2005) for more formal discussion of this subject. Calculations performed on fuzzy numbers allow us to process all of this knowledge together.

Technically, two functions defined for non-negative arguments may be introduced, $L$ and $R$, (Bandemer 2006), such that they have the unique value 1 at 0 , $L(0)=R(0)=1$, equal zero for arguments greater or equal $1, L(u)=R(u)=0$ for $u \geq 1$, and are not increasing. Then, given that core $A=\{m\}$, the membership function of a fuzzy number may be constructed using the above functions as its left and right branches

$$
\begin{array}{ll}
\mu_{A}^{l}(u)=L\left(\frac{m-u}{p_{l}}\right) & \text { for } u \leq m \\
\mu_{A}^{r}(u)=R\left(\frac{u-m}{p_{r}}\right) & \text { for } u \geq m
\end{array}
$$

where $p_{l}$ and $p_{r}$ are scale parameters, see Fig. 10. Let us denote the fuzzy number constructed this way as $A=\left(m, p_{l}, p_{r}\right)_{L R}$.

Although operations on fuzzy sets or fuzzy numbers can be defined in a more general context, they are first restricted only to fuzzy numbers described in the 
above $L R$ form. For two fuzzy numbers $A=\left(m, p_{l}, p_{r}\right)_{L R}$ and $B=\left(n, q_{l}, q_{r}\right)_{L R}$ the following operations are defined, see Dubois and Prade (1978):

1. Addition

$$
A+B=\left(m+n, p_{l}+q_{l}, p_{r}+q_{r}\right)_{L R}
$$

2. Multiplication by a positive real number $c$

$$
c A=\left(c m, c p_{l}, c p_{r}\right)_{L R}
$$

3. Multiplication by a negative real number $c$

$$
c A=\left(\mathrm{cm},|c| p_{r},|c| p_{l}\right)_{R L}
$$

with interchange of the function $L$ and $R$ in (31) and (32)

$$
\begin{aligned}
& \mu_{c A}^{l}(u)=R\left(\frac{c m-u}{|c| p_{r}}\right) \quad \text { for } u \leq c m \\
& \mu_{c A}^{r}(u)=L\left(\frac{u-c m}{|c| p_{l}}\right) \quad \text { for } u \geq c m
\end{aligned}
$$

In the general case, the interval calculus for the $\eta$-cuts can be used to obtain the appropriate operation.

\section{References}

Bandemer H (2006) Mathematics of uncertainty. In: Studies in fuzziness and soft computing, vol 189. Springer Verlag, New York

Dubois D, Prade H (1978) Operations on fuzzy numbers. Int J Syst Sci 9:613-626

Dubois D, Prade H (2005) Fuzzy intervals versus fuzzy numbers: is there a missing concept in fuzzy set theory? In: Proc. 25th Linz seminar fuzzy set theory, Linz, Austria

Gillenwater M, Sussman F, Cohen J (2007) Practical policy applications of uncertainty analysis for national greenhouse gas inventories. Water, Air \& Soil Pollution. Focus 7(4-5):451-474

Hurteaux MD, Hungate BA, Koch GW (2009) Accounting for risk in valuing forest carbon offset. Carbon Balance and Management 4:1. http://www.cbmjournal.com/content/4/1/1

Jonas M, Nilsson S (2007) Prior to economic treatment of emissions and their uncertainties under the Kyoto protocol: scientific uncertainties that must be kept in mind. Water, Air \& Soil Pollution. Focus 7(4-5):495-511

Jonas M, Gusti M, Jęda W, Nahorski Z, Nilsson S (2010) Comparison of preparatory signal detection techniques for consideration in the (post-)Kyoto policy process. Clim Change. doi:10.1007/s10584-010-9914-6

Mignone BK, Hurteau MD, Chen Y, Sohngen B (2009) Carbon offsets, reversal risk and US climate policy. Carbon Balance and Management 4:3. http://www.cbmjournal.com/content/4/1/3

Monni S, Syri S, Pipatti R, Savolainen I (2007) Extension of EU emissions trading scheme to other sectors and gases: consequences for uncertainty of total tradable amount. Water, Air \& Soil Pollution. Focus 7(4-5):529-538

Nahorski Z, Horabik J, Jonas M (2007) Compliance and emission trading under the Kyoto protocol: rules for uncertain inventories. Water, Air \& Soil Pollution. Focus 7(4-5):539-558

Nahorski Z, Horabik J (2007) Compliance and emission trading rules for uncertain estimates of inventory uncertainty. In: Proc 2nd int workshop on uncertainty in greenhouse gas inventories. IIASA, Laxenburg, pp 149-161

Nahorski Z, Horabik J (2008) Greenhouse gas emission permit trading with different uncertainties in emission sources. J Energy Eng 134(2):47-52 
Ramirez AR, de Keizer C, van der Sluijs JP (2006) Monte Carlo analysis of uncertainties in The Netherlands greenhouse gas emission inventory for 1990-2004. Report NWS-E2006-58. Copernicus Institute for Sustainable Development and Innovation. Utrecht. http:// www.chem.uu.nl/nws/www/publica/publicaties2006/E2006-58.pdf

Stern N (2007) The economics of climate change. In: The stern review. Cambridge University Press, Cambridge

Winiwarter W (2004) National greenhouse gas inventories: understanding uncertainties versus potential for improving reliability. Water, Air \& Soil Pollution. Focus 7(4-5):443-450

Winiwarter W, Muik B (2007) Statistical dependences in input data of national GHG emission inventories: effects on the overall GHG uncertainty and related policy issues. In: Presentation at 2nd int workshop uncertainty in greenhouse gas inventories, IIASA, Laxenburg, Austria, 27-28 September 2007. http://www.ibspan.waw.pl/ghg2007/Presentation/Winiwarter.pdf 